\title{
DOCTOR HONORIS CAUSA OTORGADO POR LA UNIVERSIDAD RICARDO PALMA AL DR. ALFREDO QUIÑONES-HINOJOSA
}

\author{
DOCTOR HONORIS CAUSA GRANTED BY THE RICARDO PALMA UNIVERSITY TO \\ DR. ALFREDO QUIÑONES-HINOJOSA
}

Jhony A. De La Cruz -Vargas ${ }^{1, a, b, c}$

\section{Quiero saludar en primer lugar a la Mesa de Honor:}

- Excelentísimo Dr. Elio Ivan Rodríguez Chávez, Rector de la Universidad Ricardo Palma.

- Honorable Dr. Alfredo Quiñones-Hinojosa, de quien tengo el Honor esta noche de esbozar una breve semblanza de su prolifera producción y aportes a la medicina y a la sociedad.

- Señor Secretario General de la URP, Lic. Andrés Enrique Maldonado Herrera.

- Distinguidos Vicerrectores, Decanos, Directores, Autoridades de Sociedades científicas, Hospitales e Instituciones.

- Docentes, Colegas, Estudiantes, Señoras y Señores.

El Doctor Honoris Causa, es el máximo titulo honorifico que otorga la Universidad Ricardo Palma, a personas eminentes. Son conferidos con celo por nuestra casa de estudios a muy destacadas personalidades del ámbito nacional e internacional, quienes con su obra y trayectoria personal honran a la cultura, la ciencia y la humanidad. Quiero agradecer al Rector de la Universidad por recibir el encargo, para realizar una breve semblanza del Dr. Alfredo Quiñones-Hinojosa.

México y Perú son países hermanos que comparten muchos vínculos desde tiempos inmemoriales. Ambos son paises multiculturales, plurietnicos y multilingues.

Pero ¿Porque estamos hoy en esta ceremonia donde la Universidad Ricardo Palma otorga el Doctor Honoris Causa?.

¿Que es lo mas destacable del Dr. Alfredo Quiñones-Hinojosa?:

1. ¿Su Intensa y enriquecedora aportacion cientifica?

2. ¿Su renombre académico y su trayectoria académica por las mejores universidades de EEUU?

3. ¿Su apreciada y valorada atención cotidiana como medico neurocirujano y el respeto y admiración de colegas, amigos y pacientes?

4. ¿Su prestigio como Investigador Científico?

5. ¿Su dedicación y vocación como Profesor y Mentor de muchas generaciones de medicos, residentes y pasantes de su servicio y laboratorio de investigación?

6. ¿Su admirable calidad humana, sus valores, y su interés de ayudar aun a los mas necesitados, con la creacion de la Fundación Misión: Braim?

O Quizás todo ello junto, algo no usual de observar en nuestros tiempos.

Sin lugar a dudas para llegar a donde ha llegado y estar donde el Dr. Alfredo está, ha construido su éxito desde

\footnotetext{
${ }^{1}$ Director General del INICIB, URP, Lima-Perú.

aspecialista en Oncología Medica.

' Maestría en Investigación Clínica.

Doctorado en Medicina.
}

Citar como: Jhony A. De La Cruz-Vargas. Doctor honoris causa otorgado por la Universidad Ricardo Palma al Dr. Alfredo Quiñones-Hinojosa. [Editorial]. Rev. Fac. Med. Hum. 2019;19(2):00-00. (Abril 2019). DOI 10.25176/RFMH.v19.n2.2003 
muy joven y luchando desde abajo, llegando a ser un ser humano digno de admiración.

Sus investigaciones cuentan con una extraordinaria valoración y reconocimiento en todo el mundo, con numerosos libros publicados, en varios países y varios idiomas. Cientos de artículos en las revistas de mayor impacto y prestigio, e infinidad de participaciones en congresos nacionales e internacionales.

Mas de 400 trabajos científicos publicados y otros en proceso de pubicación. Ha recibido reconociemientos y diversos premios y distinciones:

\section{DISTINCIONES ACADÉMICAS:}

\section{Títulos honoríficos:}

- 2015 / University of Notre Dame (Indiana). Doctor en ciencia: Honoris Causa

- 2016 / Loyola University (Chicago). Doctor en ciencia: Honoris Causa

- 2018 / Universidad de Santander (Colombia). Doctor de Neurociencia: Honoris Causa

\section{Profesor honorario:}

- 2008 / Universidad de las Américas, Quito, Ecuador. Profesor honorario

- 2012 / Universidad Tecnológico de Monterrey, Monterrey, México. Profesor honorario

Es miembro de otras muchas organizaciones internacionales, científicas y de investigación.

Toda una vida de incesante dedicación a la docencia e investigación, además de sus actividades clínicas, el Dr. Quiñones-Hinojosa lidera la investigación financiada por los NIH para encontrar una cura para el cáncer cerebral. Su investigación de vanguardia se centra en los tumores cerebrales y la migración celular, las disparidades en el cuidado de la salud y los resultados clínicos para pacientes neuroquirúrgicos en los que se utiliza el quirófano como una extensión de su escenario de investigación.

Ha sembrado sus conocimientos por gran parte del mundo, impartiendo cursos y conferencias, además de las múltiples misiones para tratar a personas carenciadas, como la de ayer y hoy en Lima, donde se atendieron y operaron pacientes sin costo.

Creció y estudio en Mexicalli, Baja California, México, Tras finalizar la preparatoria cuando solo tenia 19 años, tuvo que salir adelante por sus propias fuerzas, cruzando a los Estados Unidos, solo acompañado por su indudable inteligencia, su inigualable espíritu de trabajo y sin escatimar esfuerzos, ha llegado a ser una de las personalidades más reconocidas, admiradas y valoradas.

En todo momento ha dado muestras de su desinteresada entrega, cordialidad, amistad y apoyo permanente a todo el que necesita su ayuda. Para ser realmente grande hay que estar con la gente, no por encima de ella. Decía Montesquieu. Y usted Dr. Alfredo ha estado, esta y estoy seguro que estará siempre con todos los que lo necesiten ...

Cuando era niño veía a su abuela que era partera, ella ayudaba a las personas y era reconocida por la comunidad... pero eso si con carácter fuerte.... y quizás allí fue naciendo la vocación de ser medico y ayudar a las personas ... Dice un proverbio árabe “Quién no comprende una mirada, no comprenderá una larga explicación”.

Alfredo Quiñones-Hinojosa nació en un pueblo en las afueras de Mexicali, cerca de Baja California, en México. Sus primeros años transcurrieron en una pequeña comunidad agraria, donde llevó una vida humilde. "Mis padres no tenían mucho, por no decir que tenían muy poquito", dice el Doctor Q. "Pero lo que parece ser una situación difícil terminó siendo una de las bendiciones más grandes que me ha dado la vida. Por eso estoy aquí, porque sé cuáles son mis raíces, y porque mi trayectoria ha estado llena de aventuras", agrega.

"El cerebro es la frontera inexplorada. Tenemos más conexiones y sinapsis en el cerebro que estrellas en la galaxia. Es algo fascinante. De pequeñito miraba las estrellas porque nos acostábamos en el techo de nuestra casa por el calor, y soñaba, y pienso que desde entonces se me quedó esa fantasía", cuenta el Doctor Q.

En el año 1987, cuando Alfredo tenia solo 19 años de edad, decidió cruzar la frontera de México a EEUU, y que creen que paso, lo captura la MIGRA y lo envió de regreso a México, pero su persistencia hizo que inmediatamente vuelva a cruzar la frontera a EEUU. Es esta una lección de vida para los jóvenes estudiantes presentes? No existe una barrera que no puede ser superada con voluntad, trabajo y constancia. Trabajo como agricultor en cultivos de tomate. Algodón ... en el valle de Fresno, California

Hasta la fecha lleva mas de 2,000 Tumores Cerebrales operados ... (Vidas que ayudo a salvar..)

Después de UC Berkeley donde estudio Neurociencias y Psicología, llegó a la Facultad de Medicina de la Universidad de Harvard, quizás la más importante a 
nivel mundial, donde empezó a distinguirse no solo como un gran proyecto de doctor, sino también por sus labores sociales y por su capacidad de conectar con la gente. Tras su residencia posdoctoral en San Francisco, donde Quiñones-Hinojosa se convirtió ya en un neurocirujano a carta cabal, llegó a Johns Hopkins, uno de los hospitales y centros de formación de medicina más prestigiosos, donde fue profesor asociado de Cirugía Neurológica, profesor asociado de Oncología, director del Programa de Cirugía de Tumores Cerebrales en el Johns Hopkins Bayview Medical Center, y director del Programa de Cirugía Pituitaria. Actualmente ocupa la Jefatura del Departamento de Neurocirugia en la Mayo Clinic, Florida en los EEUU.

Recientemente, Disney y otras compañias productoras quieren hacer la película de la vida del Dr. Alfredo Q, en opinión del Dr. Alfredo Quiñones quizas una historia paralela a CINDERELLA en versión masculino... (Dr. Q MD). Incluso se habla de Brand Pit como el que haría el papel de Dr. Q
En la Universidad Ricardo Palma, Dr. Quiñones Hinijosa, compartimos igual que usted el sueño de que juntos podemos seguir cambiando el mundo... y si la vida lo permite estamos listos para sumar esfuerzos y colaboraciones para seguir ayudando a muchas personas...

Esta noche distinguimos y honramos a un hombre que representa a muchos latinoamericanos, que con su esfuerzo, su trabajo, su disciplina, su talento, su inteligencia, sus valores, y su espíritu de ayudar a las personas ha consagrado su vida a la labor como medico, como profesor, como investigador y para muchos como amigo Dr. Q.

\section{Muchas gracias.}

Prof. Dr. Jhony A. De La Cruz Vargas

Director del Instituto de Investigacion en Ciencias Biomedicas

Universidad Ricardo Palma.

Consulte la Versión Electrónica de la Revista:

Facultad de Medicina Humana Universidad Ricardo Palma

http://revistas.urp.edu.pe/index.php/RFMH
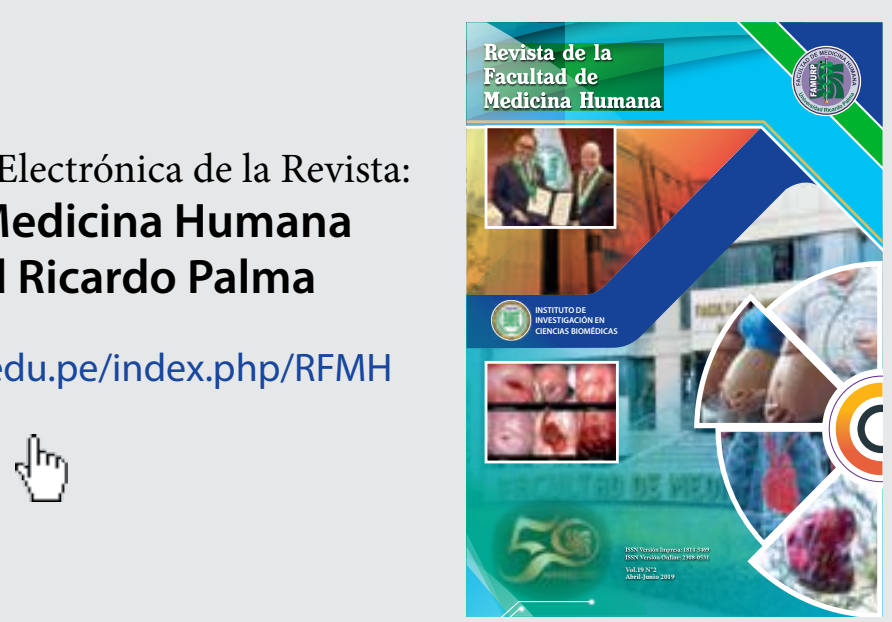

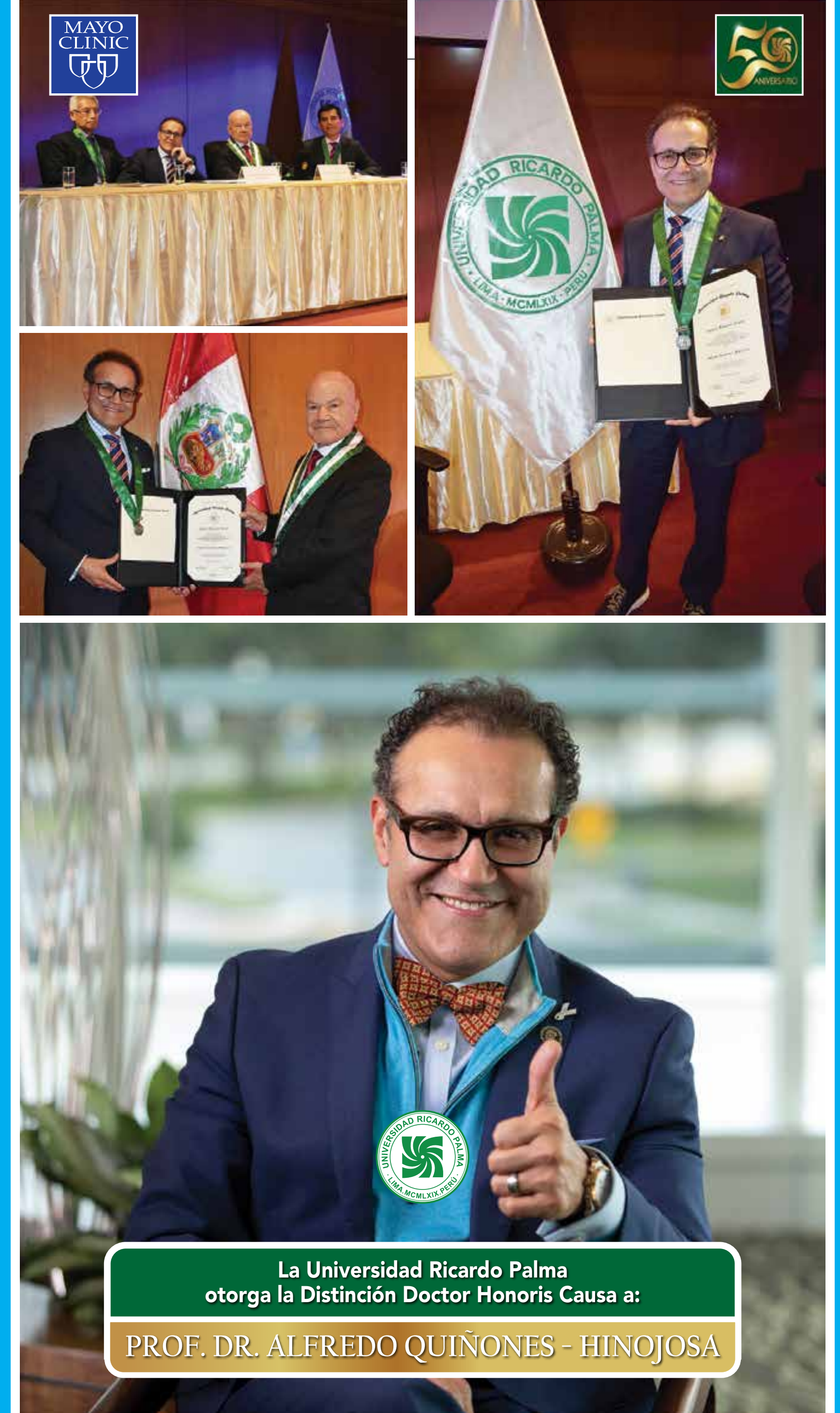